\title{
Spectral evolution of the supergiant HMXB IGR J16320-4751 along its orbit using XMM-Newton
}

\author{
Federico García ${ }^{1,2,3}$, Federico A. Fogantini ${ }^{2,3}$, Sylvain Chaty ${ }^{1}$, and Jorge A. Combi ${ }^{2,3}$ \\ ${ }^{1}$ Laboratoire AIM (UMR 7158 CEA/DRF-CNRS-Université Paris Diderot), Irfu/Département d'Astrophysique, Centre de Saclay, \\ 91191 Gif-sur-Yvette Cedex, France \\ 2 Instituto Argentino de Radioastronomía (CCT-La Plata, CONICET; CICPBA), C.C. No. 5, 1894 Villa Elisa, Argentina \\ e-mail: fgarcia@fcaglp.unlp.edu.ar \\ 3 Facultad de Ciencias Astronómicas y Geofísicas, Universidad Nacional de La Plata, Paseo del Bosque s/n, \\ 1900 La Plata, Argentina
}

Received 4 May 2018 / Accepted 10 July 2018

\begin{abstract}
Context. The INTEGRAL satellite has revealed a previously hidden population of absorbed high-mass X-ray binaries (HMXBs) hosting supergiant stars. Among them, IGR J16320-4751 is a classical system intrinsically obscured by its environment, with a column density of $\sim 10^{23} \mathrm{~cm}^{-2}$, more than an order of magnitude higher than the interstellar absorption along the line of sight. It is composed of a neutron star rotating with a spin period of $\sim 1300 \mathrm{~s}$, accreting matter from the stellar wind of an O8I supergiant star, with an orbital period of $\sim 9$ days.

Aims. We investigated the geometrical and physical parameters of both components of the binary system IGR J16320-4751. Since in systems of this type the compact object is usually embedded in the dense and powerful wind of an OB supergiant companion, our main goal here was to study the dependence of the X-ray emission and column density along the full orbit of the neutron star around the supergiant star.

Methods. We analyzed all existing archival XMM-Newton and Swift/BAT observations collected between 2003 and 2008, performing a detailed temporal and spectral analysis of the X-ray emission of the source. We then fitted the parameters derived in our study, using a simple model of a neutron star orbiting a supergiant star.

Results. The XMM-Newton light curves of IGR J16320-4751 display high-variability and flaring activity in X-rays on several timescales, with a clear spin period modulation of $\sim 1300 \mathrm{~s}$. In one observation we detected two short and bright flares where the flux increased by a factor of $\sim 10$ for $\sim 300 \mathrm{~s}$, with similar behavior in the soft and hard X-ray bands. By inspecting the 4500 -day light curves of the full Swift/BAT data, we derived a refined period of $8.99 \pm 0.01$ days, consistent with previous results. The XMMNewton spectra are characterized by a highly absorbed continuum and an Fe absorption edge at $\sim 7 \mathrm{keV}$. We fitted the continuum with a thermally comptonized COMPTT model, and the emission lines with three narrow Gaussian functions using two TBABS absorption components, to take into account both the interstellar medium and the intrinsic absorption of the system. For the whole set of observations we derived the column density at different orbital phases, showing that there is a clear modulation of the column density with the orbital phase. In addition, we also show that the flux of the Fe $\mathrm{K} \alpha$ line is correlated with the $N_{\mathrm{H}}$ column, suggesting a clear link between absorbing and fluorescent matter that, together with the orbital modulation, points towards the stellar wind being the main contributor to both continuum absorption and $\mathrm{Fe} \mathrm{K} \alpha$ line emission.

Conclusions. Assuming a simple model for the supergiant stellar wind we were able to explain the orbital modulation of the absorption column density, Fe K $\alpha$ emission and the high-energy Swift/BAT flux, allowing us to constrain the geometrical parameters of the binary system. Similar studies applied to the analysis of the spectral evolution of other sources will be useful to better constrain the physical and geometrical properties of the sgHMXB class.
\end{abstract}

Key words. X-rays: individuals: IGR J16320-4751 - stars: massive - stars: neutron - X-rays: binaries

\section{Introduction}

Since its launch in 2002, the IBIS/ISGRI detector (Lebrun et al 2003; Ubertini et al. 2003) on board the International GammaRay Astrophysics Laboratory (INTEGRAL) has discovered several sources belonging to a class of highly absorbed lowluminosity X-ray sources, which makes them difficult to detect in the soft X-ray band $(<3 \mathrm{keV})$. Most of them exhibit high levels of obscuration (e.g., Zurita Heras et al. 2006; Rodriguez et al. 2006) or extreme flaring behavior characterized by hard X-ray flux variations of several orders of magnitude on timescales of a few hours (Negueruela et al. 2006; Sguera et al. 2006). The great majority of this new kind of hard X-ray sources are of Galactic origin since INTEGRAL has spent a considerable fraction of its observational time pointing towards the Galactic plane. Observations performed with missions such as XMM-Newton or Chandra, with high astrometric accuracy at the level of the arc-second, allowed the determination of their position leading to the possible optical identification of their companion counterparts (Chaty et al. 2008; Coleiro et al. 2013). For instance, more than a dozen of these X-ray sources were detected in the direction of the Norma Galactic spiral arm and the vast majority of them are thought to be highmass X-ray binaries (HMXB) with early-type companion stars. Many of these obscured HMXBs were found to be persistent for several years showing high intrinsic variability on several 
timescales. In some cases a regular period was detected in the hard X-ray band, generally attributed to the orbital motion (Corbet 1986).

The transient hard X-ray source IGR J16320-4751 belongs to this new class of highly absorbed binary systems. The source was discovered on February 1, 2003, with the INTEGRAL observatory during ToO observations of 4U 163047 (Tomsick et al. 2003). It showed a significant variability in the $15-40 \mathrm{keV}$ energy range, being also detected in some occasions above $60 \mathrm{keV}$ (Tomsick et al. 2003; Foschini et al. 2004). Its position $\mathrm{RA}_{\mathrm{J} 2000}=16^{\mathrm{h}} 32^{\mathrm{m}}, \mathrm{Dec}_{\mathrm{J} 2000}=-47^{\mathrm{d}} 51^{\mathrm{m}}$ was coincident with the X-ray source AX J1631.9-4752, which was previously observed with the ASCA telescope (Sugizaki et al. 2001). The ASCA spectrum was modeled by a power law with a hard photon index $\Gamma \sim 0.2 \pm 0.2$ (Sugizaki et al. 2001), which suggested that the source could belong to the HMXB class.

Follow-up observations of the source with XMM-Newton on March 4, 2003, confirmed the complex temporal behavior of the source, showing several flaring events without significant variations in its hardness (Rodriguez et al. 2003). These observations improved the source position to a radius of 3 arcsec of accuracy at RA $=16^{\mathrm{h}} 32^{\mathrm{m}} 01.9^{\mathrm{s}}, \mathrm{Dec}=-47^{\circ} 52^{\prime} 27^{\prime \prime}$ (Rodriguez et al. 2003, 2006). Using the same XMM-Newton observations, Lutovinov et al. (2005) were able to unambiguously identify a pulsation period of $P=1309 \pm 40 \mathrm{~s}$, confirming the Rodriguez et al. (2003) claim about the nature of the system as an HMXB with an accreting neutron star (NS). Later on, using INTEGRAL observations, Rodriguez et al. (2006) were able to confirm the presence of pulsations above $20 \mathrm{keV}$, clearly present in the Comptonized emission realized in the close vicinity of the NS, with a pulse fraction independent of the energy. Combining the XMM-Newton and INTEGRAL spectra, Rodriguez et al. (2006) fitted an absorbed power law with $\Gamma \sim 1.6$ and a high absorption column of $N_{\mathrm{H}} \sim 2.1 \times 10^{23} \mathrm{~cm}^{-2}$, incorporating a narrow iron line at $\sim 6.4 \mathrm{keV}$. An orbital period of $8.96 \pm 0.01$ days was found from a Swift/BAT light curve extending from December 21, 2004, to September 17, 2005 (Corbet et al. 2005), and of $8.99 \pm 0.05$ days with INTEGRAL (Walter et al. 2006). The location of this system on the pulse/orbital period diagram (Corbet 1986) is typical of a NS accreting from the wind of an early supergiant companion.

Chaty et al. (2008) identified the most likely infrared counterpart (2MASS J16320215-4752289, invisible in the optical, Rodriguez et al. 2006), in agreement with Negueruela \& Schurch (2007), and rejecting other possible candidates based on a photometric analysis. In their blue nearinfrared (NIR) spectra, Chaty et al. (2008) detected only a few lines due to the high absorption, while in their red NIR spectrum they found several absorption and emission lines, such as the $\mathrm{Pa}(7-3)$ emission line, the Brackett series with $\mathrm{P}$ Cygni profiles between 1.5 and $2.17 \mu \mathrm{m}$, and He I at $2.166 \mu \mathrm{m}$, leading to the classification of the companion star as a luminous supergiant OB star, which helped to unambigously identify the stellar counterpart. Their result was also in agreement with the SED fit computed by Rahoui et al. (2008) which, including mid-IR observations, derived an optical absorption $A_{\mathrm{v}}=35.4$ mag and an O8I spectral type for the companion with $T \approx 33000 \mathrm{~K}$ and $R \approx 20 R_{\odot}$, suggesting a distance of $3.5 \mathrm{kpc}$ to the source. Finally, in a more recent study, Coleiro et al. (2013) performed NIR spectroscopy showing a faint broad He I emission and classified the stellar companion as an BN0.5 Ia supergiant star.

In this paper we report a detailed temporal and spectral analysis of nine XMM-Newton public observations of IGR J163204751. In Sect. 2 we provide details about the XMM-Newton and
Swift/BAT observations and data reduction methods that were employed for the analysis. We describe the temporal and spectral X-ray analysis and results in Sect. 3. Finally, in Sect. 4 we discuss these results in the context of a simple model developed to account for the spectral orbital variability, and we summarize our conclusions in Sect. 5.

\section{Observations and data analysis}

\subsection{XMM-Newton data}

The XMM-Newton observatory has two X-ray instruments on board: the European Photon Imaging Camera (EPIC) and the Reflecting Grating Spectrometers (RGS). EPIC consists of three detectors, two MOS cameras, MOS1 and MOS2 (Turner et al. 2001), and a PN camera (Strüder et al. 2001), which operate in the $0.3-12 \mathrm{keV}$ energy range. RGS is formed of two highresolution spectrometers working in the $0.3-2.0 \mathrm{keV}$ energy band.

IGR J16320-4751 was observed twice in March 2003 (Rodriguez et al. 2003; Lutovinov et al. 2005) and August 2004 (Rodriguez et al. 2006), and nine times between August 14 and September 17, 2008 (for a preliminary analysis, see Zurita Heras et al. 2009). The first observation was performed with a medium filter in Large Window (LW) mode, while the rest of the exposures were conducted with a thin filter in Prime Full Window observation mode. Since the PN effective area is several times larger than the MOS CCDs, and the latter were not in many of the observations, we present here the analysis of the EPIC PN data set. As RGS covers only the highly absorbed soft band up to $\sim 2.0 \mathrm{keV}$, we do not use those spectrometers in our analysis.

We reduced the XMM-Newton data by means of the Science Analysis System (SAS) version 16.0.0 and the latest calibrations available on June 2017. We obtained event lists from the PN data set after processing the Observation Data Files (ODF) with the EPPROC task. In order to exclude high-background periods we produced background light curves, excluding a circle of 100 arcsec surrounding the bright IGR J16320-4751 source, for events with energies above $10 \mathrm{keV}$. Good time intervals (GTI) were obtained excluding intervals $3 \sigma$ above the mean count rate of each light curve.

\section{Pile-up treatment}

We investigated in detail the presence of pile-up, since the average count rate in the EPIC PN camera was close to or above the level at which pile-up effects can become significant for the vast majority of the observations in the whole sample. In order to do this, we used the SAS task EPATPLOT to create diagnoses of the relative ratios of single (PATTERN==0) and double (PATTERN=[1:4]) events to study their possible deviation from the standard values expected from the PN camera calibrations. By selecting circular regions of $30^{\prime \prime}$ radii surrounding IGR J16320-4751, we found that seven of the nine series and one of the earlier observations were affected by pile-up.

Then, following the standard procedure suggested by the $X M M$-Newton calibration team, for each of the piled-up observations we extracted spectra selecting single and double events on several concentric annuli with fixed outer radius of $35^{\prime \prime}$, varying the inner radii by integer factors of the PSF: 80, 120, 160, and 200 in physical units, which correspond to $4^{\prime \prime}, 6^{\prime \prime}, 8^{\prime \prime}$, and $10^{\prime \prime}$. We thus extracted one spectrum for each of the defined regions and carefully inspected each EPATPLOT produced in order 

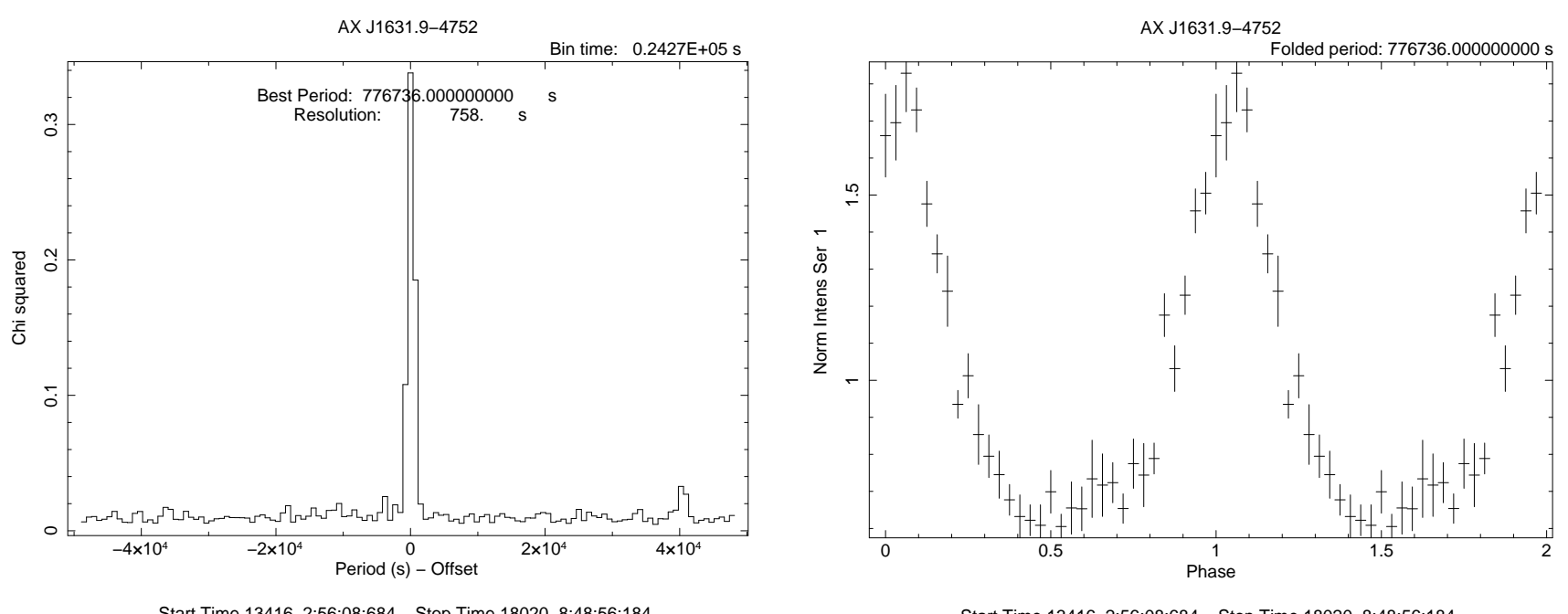

Fig. 1. Left panel: best period of 8.99 days found using the EFSEARCH of HEASOFT for the full $\sim 4500$-day Swift/BAT X-ray light curve. Right panel: folded light curve of Swift/BAT data spanning from 2004 Apr 24 to 2017 Sept 26 using the best period.

Table 1. XMM-Newton PN observations used in this work.

\begin{tabular}{|c|c|c|c|c|c|c|c|c|}
\hline ObsID & $\begin{array}{l}\text { Start date } \\
\text { (UTC) }\end{array}$ & $\begin{array}{l}\text { End date } \\
\text { (UTC) }\end{array}$ & Filter/Mode & $\begin{array}{l}\text { Exp. time } \\
(\mathrm{ks})\end{array}$ & $\begin{array}{l}\text { GTI } \\
(\mathrm{ks})\end{array}$ & $\begin{array}{c}\text { Exc. radius } \\
(\operatorname{arcsec})\end{array}$ & $\begin{array}{c}\text { Rate } \\
\left(\text { cts s }^{-1}\right)\end{array}$ & Color \\
\hline 0128531101 & $2003-03-0420: 58$ & 2003-03-05 03:12 & Medium/LW & 4.78 & 4.47 & 0 & 0.2 & 0.88 \\
\hline 0201700301 & 2004-08-19 13:28 & 2004-08-20 03:20 & Thin1/FW & 38.0 & 33.9 & 10 & 0.4 & 1.09 \\
\hline 0556140101 & $2008-08-1422: 41$ & $2008-08-1501: 12$ & Thin1/FW & 5.33 & 4.64 & 4 & 0.5 & 1.53 \\
\hline 0556150201 & $2008-08-16 \quad 17: 38$ & 2008-08-16 19:52 & Thin1/FW & 1.63 & 1.42 & 0 & 1.0 & 1.52 \\
\hline 0556140301 & $2008-08-18$ 13:33 & $2008-08-18 \quad 15: 31$ & Thin1/FW & 1.23 & 1.07 & 4 & 0.55 & 1.24 \\
\hline 0556140401 & 2008-08-20 07:34 & 2008-08-20 10:41 & Thin1/FW & 11.2 & 9.78 & 4 & 0.5 & 1.37 \\
\hline 0556140501 & 2008-08-21 07:02 & 2008-08-21 07:39 & Thin1/FW & 1.34 & 1.16 & 6 & 0.45 & 1.33 \\
\hline 0556140601 & 2008-08-22 03:54 & 2008-08-22 07:20 & Thin1/FW & 12.4 & 10.8 & 10 & 0.45 & 1.39 \\
\hline 0556140701 & $2008-08-24$ 18:28 & $2008-08-2420: 59$ & Thin1/FW & 6.03 & 5.21 & 4 & 0.4 & 2.54 \\
\hline 0556140801 & $2008-08-26$ 13:33 & 2008-08-26 16:13 & Thin1/FW & 9.63 & 8.37 & 8 & 0.4 & 1.22 \\
\hline 0556141001 & 2008-09-17 01:25 & 2008-09-17 03:31 & Thin1/FW & 4.33 & 3.77 & 0 & 0.45 & 1.38 \\
\hline
\end{tabular}

Notes. LW and FW modes correspond to PN Large Window and Full Window, respectively. Exposure time and good time intervals (GTI) are shown in ks. The excision radii used in the pile-up treatment for each observation are indicated in arcsec and the columns "Rate" and "Color" correspond to the average count rate and soft/hard color ratio in the corresponding annular extraction regions.

to define the minimum excision radii needed to avoid pile-up in each of the observations.

For subsequent analysis, events with $\mathrm{FLAG}==0$ were selected with EVSELECT task, except for the early ObsID 0128531101, for which we used the XMMEAEP selector. In Table 1 we present the whole set of XMM-Newton observations used throughout this work, including excision radii used, average count rate, and mean color.

In order to check the quality of our pile-up treatment, we produced spectra for single events (PATTERN==0), double events (PATTERN IN [1:4]), and for a combination of single and double events $($ PATTERN $<=4)$. We fitted them separately and checked that the results were consistent with each other within the uncertainties. After that successful step, for the subsequent analysis we only considered the latter set which had the best $\mathrm{S} / \mathrm{N}$.

\subsection{Swift/BAT data}

The Swift/Burst Alert Telescope (BAT) is a transient monitor that provides permanent coverage of the hard X-ray sky in the $15-50 \mathrm{keV}$ energy range. The BAT observes $\sim 90 \%$ of the sky each day with a detection sensitivity of $5.3 \mathrm{mCrab}$ in a full-day observation, with a time resolution of $64 \mathrm{~s}$ (Krimm et al. 2013). The primary interface for the BAT transient monitor is a public website $^{1}$ where more than 900 source light curves are available spanning more than eight years. Between 2005 and 2013, the monitor detected 245 sources: 146 of them being persistent and 99 seen only in outburst.

We used the full Swift/BAT data available up to September 26,2017 , in the online service ${ }^{2}$ of daily and orbital light curves to obtain a refined period of $8.99 \pm 0.01$ days, fully consistent with that found by Walter et al. (2006) with INTEGRAL and similar to the $8.96 \pm 0.01$ days found by Corbet et al. (2005) with the first year of BAT data available at that date. We divided the full 12-year span into ten intervals and checked for the consistency of the period found. In Fig. 1 we show the best-period found using the EFSEARCH task of HEASOFT and the resulting folded X-ray light curve in the $15-50 \mathrm{keV}$ band where a clear modulation of

\footnotetext{
1 https://swift.gsfc.nasa.gov/results/transients/ 2 https://swift.gsfc.nasa.gov/results/transients/ AXJ1631.9-4752/
} 
0556140801 Light-Curves bin time: $20 \mathrm{~s}$
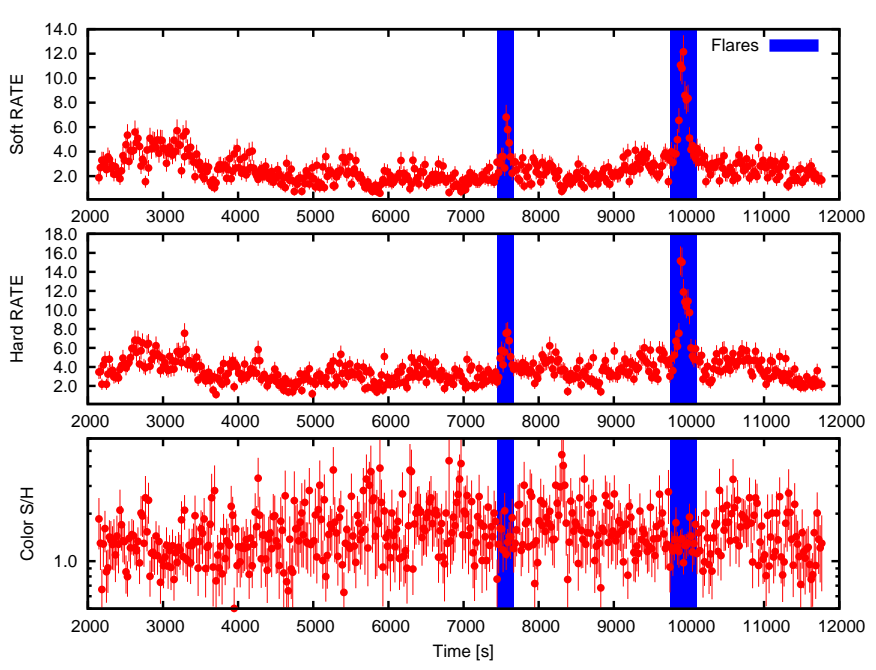

Fig. 2. XMM-Newton PN background-corrected light curves of observation 0556140801 of IGR J16320-4751 using 20 s binning time. Top panel: soft band $(0.5-6.0 \mathrm{keV})$ rate in $\mathrm{cts} \mathrm{s}^{-1}$. Central panel: hard band $(6.0-12.0 \mathrm{keV})$ rate in $\mathrm{cts} \mathrm{s}^{-1}$. Bottom panel: soft-to-hard color ratio. Blue stripes correspond to two flaring intervals where the rate significantly increases in both soft and hard bands, keeping an average color value consistent with the rest of the observation. Error bars correspond to the $1-\sigma$ confidence level.

$0.6-1.8 \mathrm{cts} \mathrm{s}^{-1}$ was obtained for the full $\sim 4500$-day light curve. The period reference corresponds to MJD 54702.82292, fixed at the middle of ObsID 0556140701 from the XMM-Newton campaign.

\section{Results}

\subsection{XMM-Newton light curves}

We extracted barycenter-corrected light curves using the annular regions determined by the pile-up analysis with an outer radius of $35^{\prime \prime}$. For background regions we selected circles of $50^{\prime \prime}$ in the same CCD of the source as suggested in the XMM-Newton Calibration Notes ${ }^{3}$. Background-subtracted and exposure-corrected light curves were calculated using the EPICLCCORR SAS task.

Considering a total average count rate of $3.56 \mathrm{cts} \mathrm{s}^{-1}$, we chose a binning time of $20 \mathrm{~s}$ in order to analyze the X-ray variability and flaring activity of the source. Moreover, considering the overall shape of the spectra, we decided to use two energy bands to analyze the source color evolution: a soft band in the $0.5-6.0 \mathrm{keV}$ energy range and a hard band in the $6.0-12.0 \mathrm{keV}$ energy range. Then, by means of the LCURVE task from HEASOFT we created soft, hard, and soft/hard ratio light curves that we used to search for long-term variability, short flaring, and possible spectral changes of the source.

Our visual inspection of the light curves indicated high variability on several timescales, including an overall change that can be seen on the variable net count rate in Table 1. In some of them a clear modulation can be seen on a timescale of $\sim 1300 \mathrm{~s}$, as was first pointed out by Lutovinov et al. (2005), a period that was attributed to the spin of the NS in the system. This modulation is evident in ObsIDs 0128531101, 0201700301, 0556140401, and 0556140601, both in the soft and hard bands,

\footnotetext{
3 http://xmm2.esac.esa.int/docs/documents/CAL-TNQ018.pdf
}

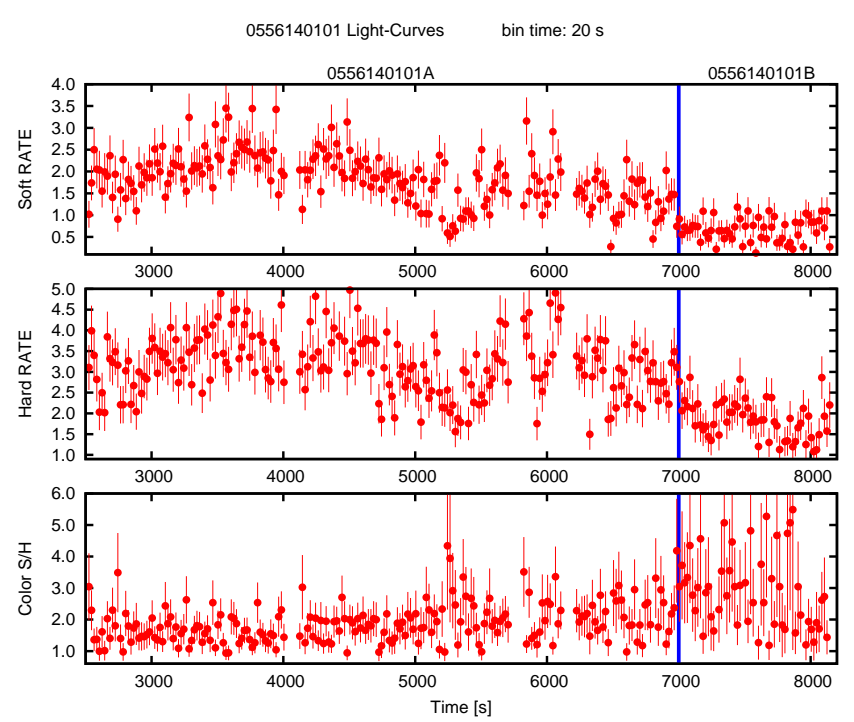

Fig. 3. XMM-Newton PN background-corrected light curves of observation 0556140101 of IGR J16320-4751 using 20 s binning time. Top panel: soft band $(0.5-6.0 \mathrm{keV})$ rate in $\mathrm{cts} \mathrm{s}^{-1}$. Central panel: hard band $(6.0-12.0 \mathrm{keV})$ rate in $\mathrm{cts} \mathrm{s}^{-1}$. Bottom panel: soft/hard color ratio. The blue lines indicate a significant variation in the source state at $\sim 7000 \mathrm{~s}$ both in the count-rates and in the color ratio. Error bars correspond to $1-\sigma$ confidence level.

keeping the color ratio constant. This modulation persists in the rest of the observations, but it is not that clear. ObsIDs 0556140401 and 0556140601 show a secular increase in their count rates, evidencing the presence of a long-term variability of the source on a timescale of $10 \mathrm{ks}$. In ObsID 0556140801, two short and bright flares were detected when the source increases its rate by a factor of $\sim 10$ for $\sim 300 \mathrm{~s}$, keeping a constant color ratio (see Fig. 2). For subsequent spectral analysis, we thus split this observation into $0556140801 \mathrm{NF}$ for the non-flaring intervals and $0556140801 \mathrm{~F}$ for the flaring ones (indicated with blue bands in Fig. 2). Even though the color ratio is $\sim 1.2-1.4$ through all the observations, two strong variations can be seen in ObsIDs 0556140101 and 0556140701 where the ratio changes from $\sim 1.5$ to $\sim 3$ in the first case and from $\sim 4$ to $\sim 2$ in the second. We decided to split these observations, naming them $\mathrm{A}$ and $\mathrm{B}$ as indicated by the blue lines in Figs. 3 and 4. In Table 1 we present the average count rates and colors for each XMM-Newton observation.

The similar behavior observed in both the soft and hard $\mathrm{X}$-ray light curves is the reason why the hardness ratio does not show significant variations between the flaring and non-flaring periods. This indicates that flares are related to a broadband flux increase and not to variations in the absorption. In this case, the hard band should be much less affected than the soft band, thus implying changes in the hardness evolution.

\subsection{XMM-Newton spectra}

We extracted source and background spectra from the same regions indicated in the light curve analysis. Redistribution response matrices (RMF) and ancillary response files (ARF) were generated using RMFGEN and ARFGEN tasks, respectively, considering the $p s f$ function. We binned the spectra to obtain at least 25 counts per bin and we fitted them with XSPEC v12.9.1 (Arnaud 1996) considering the full 0.5-12.0 keV energy band. 


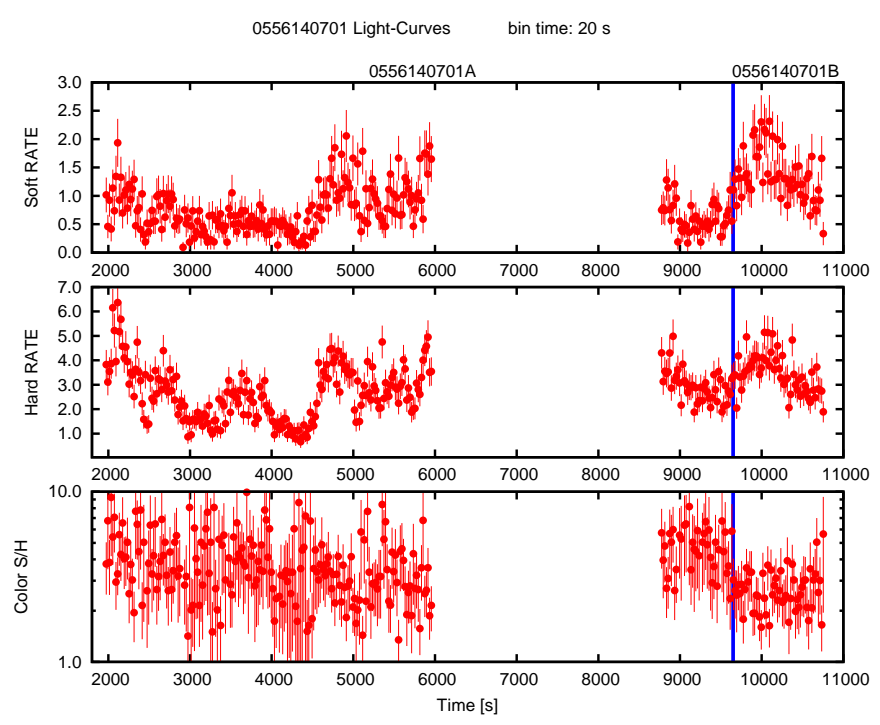

Fig. 4. Same as Fig. 3, but for observation 0556140701.

The spectra of IGR J16320-4751 are characterized by a highly absorbed continuum and a clear Fe-edge of absorption at $\sim 7 \mathrm{keV}$, which varies significantly among the different observations. In ObsID 0556140401 , only $0.4 \%$ of the counts are in the $0.5-2.0 \mathrm{keV}$ band, and this fraction decreases to $0.3 \%$ and $0 \%$ for ObsIDs 0556140701A and 0556140701B, respectively. The continuum has a power law-like shape with a highenergy cutoff that becomes evident at energies above $7-9 \mathrm{keV}$. Prominent $\mathrm{Fe}-\mathrm{K} \alpha$ lines at $\sim 6.4 \mathrm{keV}$ are present in all the spectra. In the best-quality spectra, fainter $\mathrm{Fe}-\mathrm{K} \beta$ (at $\sim 7 \mathrm{keV}$ ) and Fe XXV (at $\sim 6.7 \mathrm{keV}$ ) lines can also be seen. We fitted the spectra by means of a thermally Comptonized model COMPTT (Titarchuk 1994) for the continuum and three narrow Gaussian functions (with null widths $\sigma=0 \mathrm{keV}$ ) to model the emission lines. COMPTT is an analytical model that describes the Comptonization of soft photons with temperature $k T_{0}$ by a hot plasma with temperature $k T_{\mathrm{e}}$ and optical depth $\tau$. We included two TBABS absorption components using abundances from Wilms et al. (2000) to attain for both the interstellar medium (ISM) and the intrinsic absorption of the obscured HMXB system. We fixed the hydrogen column density of the ISM absorption model to $2.1 \times 10^{22} \mathrm{~cm}^{-2}$ as in Rodriguez et al. (2003) and we let the second hydrogen column $N_{\mathrm{H}}$ to freely vary during the fits.

We estimated confidence regions for all parameters at $90 \%$ level using the Markov chain Monte Carlo (MCMC) method implemented in XSPEC. For our calculations we ran eight walkers for a total of $8 \times 10^{4}$ steps to find the best-fit values of the free parameters together with their confidence regions (see Tables 2 and 3) as well as the reduced $\chi_{v}^{2}$ and the degrees of freedom (d.o.f.). We used the CFLUX convolution model to estimate the unabsorbed flux of the continuum Comptonization in the soft, hard, and total (0.5-12.0 keV) bands. Shortened ObsIDs are shown in the "Name" column. Phases correspond to an orbital period of 8.99 days and a central epoch corresponding to the middle of the 0556140701 exposure (phase $=0.5$ ). Phases are centered on each observation and their symmetrical error bars correspond to their respective duration. In Fig. 5, we show two examples of the XMM-Newton PN spectra of IGR J16320-4751. In the left (right) panel we show background-subtracted X-ray spectra of ObsID 401 (701). Errors are at 1- $\sigma$ confidence levels, and $\chi^{2}$ statistics are used.
The good quality of the fits obtained with the COMPTT model suggests that the continuum emission is compatible with a Comptonization of soft photons, emitted close to the surface of the NS by a diluted cloud of hot electrons surrounding the compact object. However, as first noticed by Rodriguez et al. (2003), the lack of data above $12 \mathrm{keV}$, does not allow us to obtain a good constraint on the cutoff energy. Regarding the intrinsic absorption column density, two important facts should be noted. First, in the majority of the observations, we obtain $N_{\mathrm{H}} \sim 20-30 \times 10^{22} \mathrm{~cm}^{-2}$, which is fully compatible with the values found by Rodriguez et al. (2006) with XMM-Newton and in 't Zand et al. (2003) in a BeppoSAX spectrum. Second, in Obs 101A, 101B, 701A, and 701B, we found significantly higher values of $N_{\mathrm{H}} \sim 35-60 \times 10^{22} \mathrm{~cm}^{-2}$ without noticing strong changes in the continuum emission parameters, which suggests that this variation is a geometrical effect instead of a local sudden change in the accretion process.

Despite the good quality of the fits, a systematic trend to a soft excess is suggested by the spectra (see Fig. 5). In order to test for possible systematics that could make us overpredict the $N_{\mathrm{H}}$ column value, we did two different tests. First, we added a blackbody to account for a possible soft-excess origin and fitted the spectra again, re-calculating the $90 \%$ confidence region for the $N_{\mathrm{H}}$ value. For ObsID 0401, we obtained (28-30.7) $\times$ $10^{22} \mathrm{~cm}^{-2}$, which is fully consistent with the value reported in Table 2. For ObsID 0701A, we found (57.9-71.7) $\times 10^{22} \mathrm{~cm}^{-2}$, which is consistent in the lower bound and increases for the higher bound, due to a correlation between the $N_{\mathrm{H}}$ and the blackbody. As a second test, we fitted the same spectra restricting ourselves to the $2-12 \mathrm{keV}$ energy band. For $0701 \mathrm{~A}$, we found that the $N_{\mathrm{H}}$ column is within the $(54.3-60.9) \times 10^{22} \mathrm{~cm}^{-2}$ range, while for 0401 it remains in the $(28.4-30.5) \times 10^{22} \mathrm{~cm}^{-2}$ range, being highly consistent with the values presented in Table 2 for the full $0.5-12 \mathrm{keV}$ spectral range.

Following the conversion expression $N_{\mathrm{H}}\left[\mathrm{cm}^{-2}\right]=(2.21 \pm$ $0.09) \times 10^{21} A_{\mathrm{V}}$ [mag] (Güver \& Ozel 2009), and considering the minimum X-ray column of $N_{\mathrm{H}}=2.1 \times 10^{23} \mathrm{~cm}^{-2}$ found in our series of spectra, we deduce an optical extinction of $A_{\mathrm{V}} \sim 100 \mathrm{mag}$, which is much higher than the optical extinction derived in the optical and the infrared bands (Chaty et al. 2008; Coleiro et al. 2013). In the above-mentioned papers, the authors report corresponding absorption columns of 2.14 and $6.60 \times 10^{20} \mathrm{~cm}^{-2}$ in the optical and IR bands, respectively. This difference in the absorption columns can be explained by stratification, where the observed radiation at different wavelengths originates at different depths in the system, and thus transverses different optical paths, probing different emission and absorption layers. While in the optical/IR band we measure the absorption of optically thick material down to the surface of the supergiant star, in the X-ray band we measure the intrinsic absorption down to the surface of the compact object, which seems to be embedded in a dense cloud of accreting material (similar to the case of IGR J16318-4848, Chaty \& Rahoui 2012).

While the $\mathrm{Fe} \mathrm{K} \alpha$ and $\mathrm{Fe} \mathrm{K} \beta$ lines are clearly noticeable in all the X-ray spectra, Fe XXV is not that prominent, being hardly detectable in some of the observations. In the cases where the spectral fits were not sensitive to the addition of this third Gaussian (e.g., 101B, 301, 801F), we decided to fix its normalization to zero during the fits (indicated by $\dagger$ in Table 3 ). It is important to note that in all cases we were able to obtain very good fits to the emission line by means of zero-width Gaussians, meaning that the broadening of the Fe lines (when present) should be lower than the width of the response of the PN camera at those energies. 
Table 2. Best-fit spectral parameters for the continuum emission.

\begin{tabular}{|c|c|c|c|c|c|c|c|c|c|c|}
\hline ObsID & Phase & Name & $N_{\mathrm{H}}$ & $k T_{0}$ & $k T_{\mathrm{e}}$ & $\tau$ & Norm & Flux $_{\mathrm{T}}$ & Flux $_{S}$ & Flux $_{H}$ \\
\hline 0128531101 & - & 1285 & $22.4_{-1.1}^{+0.9}$ & $<26.7$ & $2.56_{-0.11}^{+0.13}$ & $14.1_{-1.8}^{+1.7}$ & $7.7_{-1.7}^{+3.1}$ & $2.8_{-0.6}^{+0.7}$ & $1.3_{-0.4}^{+0.6}$ & $1.5_{-0.1}^{+0.1}$ \\
\hline 0201700301 & - & 2017 & $18.9_{-0.6}^{+0.1}$ & $<76.3$ & $2.77_{-0.06}^{+0.03}$ & $17.9_{-0.4}^{+1.6}$ & $16.1_{-2.1}^{+0.3}$ & $13.4_{-0.5}^{+0.5}$ & $5.2_{-0.4}^{+0.4}$ & $8.2_{-0.1}^{+0.1}$ \\
\hline 0556140101A & $0.407 \pm 0.005$ & $101 \mathrm{~A}$ & $37.6_{-1.1}^{+0.9}$ & $71.0_{-2.8}^{+2.9}$ & $3.47_{-0.22}^{+0.27}$ & $14.1_{-1.7}^{+2.8}$ & $13.0_{-1.5}^{+1.1}$ & $20.3_{-1.6}^{+1.9}$ & $7.6_{-1.3}^{+1.5}$ & $12.7_{-0.4}^{+0.4}$ \\
\hline 0556140101B & $0.412 \pm 0.001$ & 101B & $47.2_{-2.0}^{+5.6}$ & $71.1_{-16.7}^{+6.0}$ & $2.96_{-0.16}^{+0.27}$ & $100.1_{-64.9}^{+22.0}$ & $4.1_{-0.2}^{+1.0}$ & $9.6_{-0.7}^{+0.3}$ & $2.5_{-0.4}^{+1.3}$ & $7.2_{-0.5}^{+0.6}$ \\
\hline 0556140201 & $0.606 \pm 0.005$ & 201 & $26.8_{-0.6}^{+1.1}$ & $71.0_{-3.5}^{+6.2}$ & $4.91_{-0.07}^{+0.11}$ & $12.4_{-0.5}^{+0.3}$ & $18.8_{-0.4}^{+0.6}$ & $35.7_{-2.0}^{+2.1}$ & $12.4_{-16}^{+1.5}$ & $23.5_{-0.6}^{+0.6}$ \\
\hline 0556140301 & $0.809 \pm 0.005$ & 301 & $21.6_{-1.5}^{+3.1}$ & $71.0_{-10.8}^{+1.5}$ & $2.85_{-0.12}^{+0.14}$ & $35.9_{-12.1}^{+7.8}$ & $7.6_{-0.5}^{+1.8}$ & $15.5_{-1.1}^{+2.7}$ & $4.5_{-1.1}^{+2.2}$ & $10.9_{-0.5}^{+0.7}$ \\
\hline 0556140401 & $0.006 \pm 0.007$ & 401 & $29.3_{-0.4}^{+0.5}$ & $71.0_{-0.5}^{+0.9}$ & $3.33_{-0.05}^{+0.08}$ & $15.7_{-0.6}^{+0.4}$ & $13.3_{-0.2}^{-0.3}$ & $21.7_{-0.9}^{+1.0}$ & $7.8_{-0.8}^{+0.8}$ & $14.0_{-0.2}^{+0.3}$ \\
\hline 0556140501 & $0.109 \pm 0.001$ & 501 & $31.7_{-2.7}^{+5.4}$ & $71.0_{-8.3}^{+56.1}$ & $3.15_{-0.22}^{+0.84}$ & $14.2_{-5.9}^{+3.9}$ & $12.2_{-2.0}^{+2.6}$ & $17.3_{-3.3}^{+3.5}$ & $6.9_{-2.6}^{+2.9}$ & $10.4_{-0.8}^{+0.7}$ \\
\hline 0556140601 & $0.212 \pm 0.008$ & 601 & $25.8_{-0.3}^{+0.3}$ & $70.9_{-0.1}^{+0.1}$ & $3.09_{-0.04}^{+0.05}$ & $20.3_{-0.5}^{+0.5}$ & $24.7_{-0.2}^{+0.1}$ & $43.8_{-1.6}^{+2.3}$ & $14.5_{-1.4}^{+1.6}$ & $29.6_{-0.5}^{+0.5}$ \\
\hline 0556140701A & $0.499 \pm 0.005$ & $701 \mathrm{~A}$ & $58.6_{-1.2}^{+1.8}$ & $70.9_{-4.0}^{+3.6}$ & $3.84_{-0.05}^{+0.05}$ & $39.0_{-1.3}^{+0.7}$ & $6.8_{-0.1}^{+0.2}$ & $16.8_{-1.1}^{+1.7}$ & $3.6_{-05}^{+1.1}$ & $13.2_{-0.5}^{+0.5}$ \\
\hline 0556140701B & $0.505 \pm 0.001$ & 701B & $58.7_{-5.8}^{+4.0}$ & $71.0_{-4.2}^{+21.0}$ & $7.44_{-1.62}^{+0.63}$ & $9.5_{-0.3}^{+2.0}$ & $13.9_{-2.0}^{+2.6}$ & $28.6_{-4.1}^{+3.7}$ & $10.8_{-2.9}^{+2.6}$ & $17.8_{-1.2}^{+1.2}$ \\
\hline $0556140801 \mathrm{~F}$ & $0.700 \pm 0.001$ & $801 \mathrm{~F}$ & $30.2_{-2.0}^{+3.3}$ & $301.4_{-205.3}^{+416.6}$ & $3.88_{-0.02}^{+0.49}$ & $10.4_{-1.9}^{+1.5}$ & $20.2_{-3.9}^{+2.5}$ & $38.5_{-6.9}^{+6.6}$ & $16.5_{-5.4}^{+5.5}$ & $22.0_{-1.7}^{+1.5}$ \\
\hline $0556140801 \mathrm{NF}$ & $0.700 \pm 0.006$ & $801 \mathrm{NF}$ & $22.9_{-0.3}^{+0.6}$ & $71.0_{-2.0}^{+0.9}$ & $2.72_{-0.03}^{+0.03}$ & $31.8_{-1.4}^{+1.1}$ & $8.6_{-0.1}^{+0.2}$ & $16.3_{-1.0}^{+1.4}$ & $5.0_{-0.5}^{+1.2}$ & $11.1_{-0.3}^{+0.1}$ \\
\hline 0556141001 & $0.090 \pm 0.005$ & 1001 & $25.2_{-1.0}^{+0.0}$ & $71.0_{-1.9}^{+1.6}$ & $3.52_{-0.14}^{+0.17}$ & $16.1_{-1.5}^{+-1.4}$ & $13.6_{-1.0}^{-0.1}$ & $23.9_{-1.3}^{+1.4}$ & $8.2_{-1.1}^{+0.0}$ & $15.8_{-0.4}^{+0.3}$ \\
\hline
\end{tabular}

Notes. Intrinsic absorption column density, $N_{\mathrm{H}}$, is indicated in units of $10^{22} \mathrm{~cm}^{-2}$. Plasma and electron temperatures, $k T_{0}$ and $k T_{\mathrm{e}}$, are shown in $\mathrm{eV}$ and $\mathrm{keV}$, respectively. Optical depth, $\tau$, and normalization, Norm, correspond to COMPTT model. Flux $\mathrm{T}_{\mathrm{T}}(0.5-12 \mathrm{keV})$, Flux $\mathrm{S}(0.5-6 \mathrm{keV})$, and Flux $_{\mathrm{H}}(6-12 \mathrm{keV})$ are the unabsorbed X-ray fluxes in $10^{-11} \mathrm{erg} \mathrm{cm}^{-2} \mathrm{~s}^{-1}$.

Table 3. Best-fit spectral parameters of the Gaussian emission lines.

\begin{tabular}{|c|c|c|c|c|c|c|c|}
\hline ObsID & $E_{\mathrm{K} \alpha}$ & Flux $_{K \alpha}$ & $E_{\mathrm{XXV}}$ & Flux $_{X X V}$ & $E_{\mathrm{K} \beta}$ & Flux $_{K \beta}$ & $\chi_{v}^{2} /$ d.o.f. \\
\hline 0128531101 & $6.40_{-0.05}^{+0.16}$ & $1.5_{-0.7}^{+0.7}$ & $6.68^{\dagger}$ & $0.00^{\dagger}$ & $7.19_{-0.24}^{+0.04}$ & $0.96_{0.00}^{+0.14}$ & $0.84 / 70$ \\
\hline 0201700301 & $6.42_{-0.01}^{+0.01}$ & $17.7_{-0.6}^{+0.2}$ & $6.72_{-0.05}^{+0.06}$ & $3.59_{-0.08}^{+0.03}$ & $7.06_{-0.06}^{-0.05}$ & $3.80_{-0.03}^{+0.07}$ & $1.17 / 150$ \\
\hline 0556140101A & $6.42_{-0.01}^{+0.01}$ & $48.1_{-3.2}^{+3.4}$ & $6.68^{\dagger}$ & $2.96_{-0.31}^{+0.39}$ & $7.04_{-0.05}^{+0.04}$ & $6.84_{-1.07}^{+0.07}$ & $1.03 / 108$ \\
\hline 0556140101B & $6.41_{-0.03}^{+0.02}$ & $51.1_{-7.6}^{+5.4}$ & $6.68^{\dagger}$ & $0.00^{\dagger}$ & $7.05_{-0.06}^{+0.07}$ & $11.93_{-4.15}^{+3.76}$ & $0.51 / 38$ \\
\hline 0556140201 & $6.42_{-0.01}^{+0.01}$ & $66.0_{-4.7}^{+4.1}$ & $6.73_{-0.04}^{+0.07}$ & $10.30_{-1.69}^{+0.65}$ & $7.01_{-0.05}^{+0.06}$ & $14.23_{-3.63}^{+0.92}$ & $1.08 / 112$ \\
\hline 0556140301 & $6.41_{-0.03}^{+0.03}$ & $32.7_{-5.2}^{+3.8}$ & $6.68^{\dagger}$ & $0.00^{\dagger}$ & $6.97_{-0.04}^{+0.11}$ & $7.90_{-2.68}^{+1.79}$ & $1.11 / 78$ \\
\hline 0556140401 & $6.41_{-0.01}^{+0.01}$ & $35.6_{-1.5}^{+1.3}$ & $6.66_{-0.03}^{+0.03}$ & $6.66_{-0.07}^{+0.05}$ & $7.01_{-0.03}^{+0.04}$ & $8.48_{-0.30}^{+0.30}$ & $1.22 / 134$ \\
\hline 0556140501 & $6.43_{-0.05}^{+0.02}$ & $23.8_{-6.4}^{+8.6}$ & $6.68^{\dagger}$ & $0.008_{-0.003}^{+0.003}$ & $6.90_{0.01}^{+0.26}$ & $9.16_{-0.09}^{+3.12}$ & $0.77 / 66$ \\
\hline 0556140601 & $6.41_{-0.01}^{+0.01}$ & $101.7_{-0.9}^{+0.5}$ & $6.73_{-0.04}^{+0.02}$ & $14.26_{-0.31}^{+0.26}$ & $7.05_{-0.01}^{+0.01}$ & $30.88_{-0.91}^{+0.88}$ & $1.08 / 140$ \\
\hline 0556140701A & $6.41_{-0.01}^{+0.01}$ & $81.9_{-3.6}^{+3.4}$ & $6.68_{-0.04}^{+0.03}$ & $10.74_{-0.28}^{+0.09}$ & $7.01_{-0.02}^{+0.01}$ & $21.08_{-0.45}^{+0.30}$ & $1.00 / 94$ \\
\hline 0556140701B & $6.39_{-0.01}^{+0.01}$ & $85.8_{-11.2}^{+11.1}$ & $6.75_{-0.12}^{-0.04}$ & $11.29_{-0.71}^{-3.28}$ & $7.02^{-0.02}$ & $1.77_{-0.55}^{-1.45}$ & $1.07 / 66$ \\
\hline $0556140801 \mathrm{~F}$ & $6.44_{-0.01}^{+0.01}$ & $68.2_{-3.2}^{+7.2}$ & $6.68^{\dagger}$ & $0.00^{\dagger}$ & $7.02^{\dagger}$ & $0.00^{\dagger}$ & $1.00 / 57$ \\
\hline $0556140801 \mathrm{NF}$ & $6.40_{-0.01}^{+0.01}$ & $38.6_{-1.5}^{+1.8}$ & $6.63_{-0.02}^{+0.06}$ & $9.69_{-0.16}^{+0.14}$ & $6.97_{-0.03}^{+0.03}$ & $11.21_{-0.40}^{+0.41}$ & $1.28 / 119$ \\
\hline 0556141001 & $6.41_{-0.02}^{+0.01}$ & $36.8_{-0.8}^{+1.3}$ & $6.68_{-0.04}^{+0.02}$ & $4.74_{-0.09}^{-0.10}$ & $7.03_{-0.04}^{+0.03}$ & $9.47_{-0.18}^{+0.40}$ & $1.30 / 127$ \\
\hline
\end{tabular}

Notes. $E_{\mathrm{K} \alpha}, E_{\mathrm{XXV}}$, and $E_{\mathrm{K} \beta}$ are the central energies of the lines expressed in $\mathrm{keV}$ and Flux $\mathrm{K}_{\alpha}$, Flux $\mathrm{XXV}$, and Flux $\mathrm{K}_{\mathrm{K} \beta}$ are their corresponding fluxes in units of $10^{5}$ photons $\mathrm{cm}^{-2} \mathrm{~s}^{-1}$. The $\dagger$ symbol indicates parameters that were fixed during the fits.

As previously noted by Giménez-García et al. (2015), we also found a correlation between the flux of the $\mathrm{Fe} \mathrm{K} \alpha$ line and the continuum level, characterized by the total unabsorbed flux in the $0.5-12.0 \mathrm{keV}$ energy band (see left panel of Fig. 6). This is expected for fluorescence emission from a small region of matter in the very close vicinity of the illuminating compact object. We also confirm a correlation between the X-ray luminosity and the $\mathrm{Fe} \mathrm{K} \alpha$ flux, normalized by the X-ray flux, known as Baldwin effect. As pointed out by Giménez-García et al. (2015), $\gamma$ Cas sources do not follow this correlation, suggesting a different emission scenario in sgHMXBs like IGR J16320-4751.

\section{Discussion}

In Fig. 7 we show the up-to-date Corbet diagram (Corbet 1986) including all the well-known HMXBs hosting NSs. With an orbital period of $\sim 9$ days, and a quite slowly rotating NS with spin period of $\sim 1300 \mathrm{~s}$, IGR J16320-4751 is a prototype of the sgHMXB class, as shown by the pulse-orbital period diagram. The measured X-ray column density, $N_{\mathrm{H}} \approx 2 \times 10^{23} \mathrm{~cm}^{-2}$, is an order of magnitude higher than the absorption column density along the line of sight, favoring the fact that this source belongs to the intrinsically absorbed sgHMXB class. 
055640401 PN spectrum of IGR J16320-4751

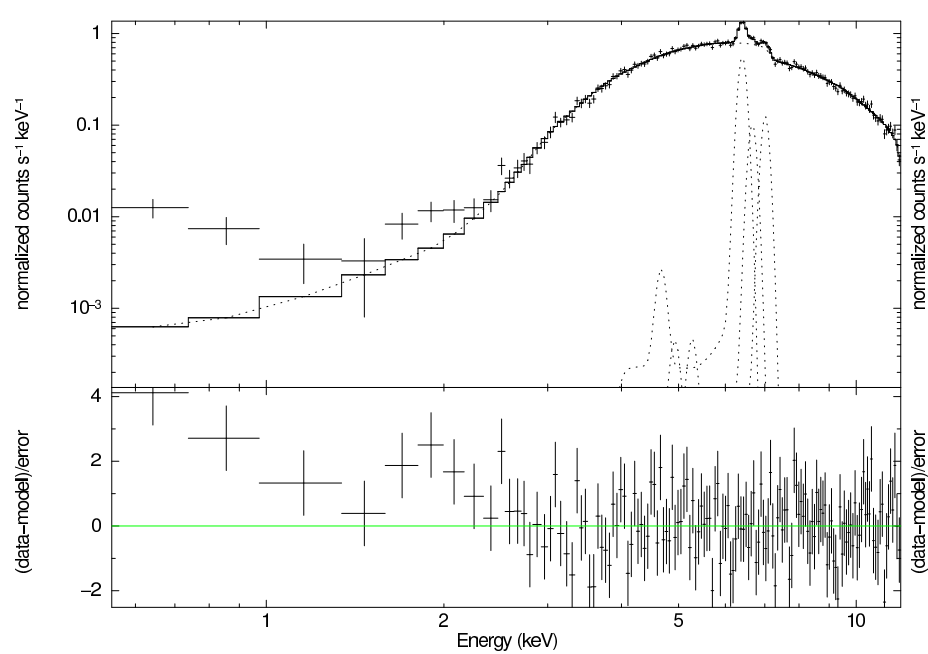

055640701 PN spectrum of IGR J16320-4751

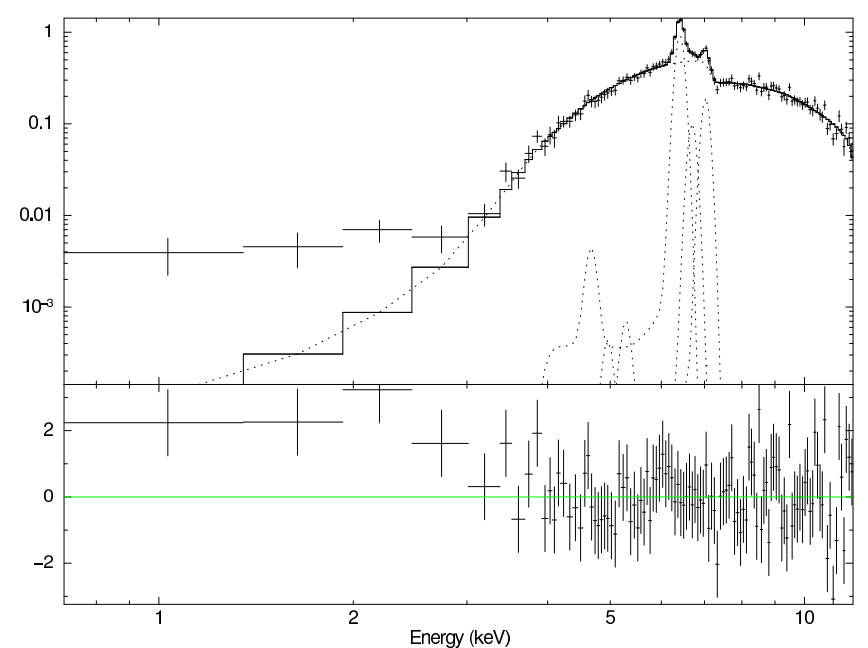

Fig. 5. XMM-Newton PN spectra and best-fit model of IGR J16320-4751 in two extreme cases. Left panel: background-subtracted X-ray spectra of ObsID 401. Right panel: background-subtracted X-ray spectra of ObsID 701. Lower panels: fitting residuals. Errors are at 1- $\sigma$ (68\%) confidence levels, and $\chi^{2}$ statistics are used.
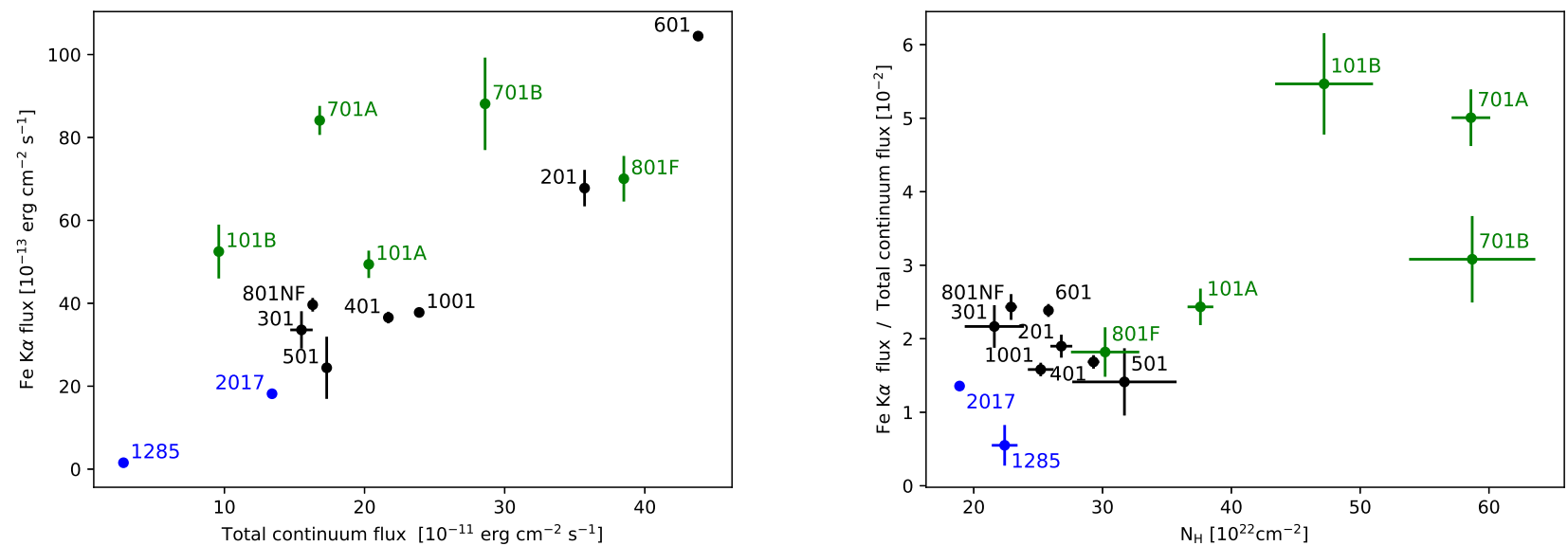

Fig. 6. Correlations between spectral parameters of IGR J16320-4751. Left panel: Flux $\mathrm{K}_{\mathrm{K} \alpha}$ with respect to Flux $\mathrm{T}_{\mathrm{T}}$. Right panel: Flux $\mathrm{K}_{\mathrm{K} \alpha}$ normalized to Flux $_{\mathrm{T}}$, with respect to the intrinsic $N_{\mathrm{H}}$. Error bars correspond to the $90 \%$ confidence level. Blue dots correspond to earlier observations from 2003 and 2004. Green dots indicate split observations (see Sect. 3.1). The figures suggest two possible correlations between these spectral parameters for the whole set of XMM-Newton observations. Using a Pearson test we found $R=0.76$ with a $p$-value $=0.002$ for the left panel and $R=0.77$ for a probability $p$-value $=0.001$ for the right panel.

In sgHMXBs like IGR J16320-4751, the observed X-ray luminosity is powered by wind-accretion onto the NS. The compact object is usually embedded in a dense and powerful wind provided by the OB supergiant companion. The X-ray emission properties directly depend on both the wind geometry (velocity and density profile, inhomogeneities, etc.) and the orbit (semiaxis and eccentricity) which together determine the wind accretion rate. Supergiant stars studies show that these extended stellar winds are quite complex. The wind has a high density-gradient decreasing outwards from the star (CAK model, Castor et al. 1975), and local random inhomogeneities or clumps are expected as an intrisic feature of the radiatively driven winds (Oskinova et al. 2012). While the latter can be responsible for short flares like those found in ObsID 801, the orbital modulation of the X-ray spectra and the hard X-ray light curve could be explained by a simple geometrical model taking into account the wind density profile and the orbital geometry with respect to a distant observer.
With the aim of better understanding the orbital modulations found in the intrinsic absorption column density and the BAT hard X-ray light curve we propose a simple model consisting of a NS orbiting an OB supergiant embedded in its dense wind. Assuming a supergiant donor with $M_{\star}=25 M_{\odot}$ and $R_{\star}=$ $20 R_{\odot}$ and a canonical NS of $1.4 M_{\odot}$, for a $P=8.99 d$, using Kepler's third law, we obtain a semimajor axis of $a=0.25 \mathrm{AU}$ which represents $\sim 2.7 R_{\star}$. We model the supergiant wind profile by means of a typical CAK model with a mass-loss rate of $3 \times 10^{-6} M_{\odot} \mathrm{yr}^{-1}, \beta=0.85$ and $v_{\infty}=1300 \mathrm{~km} \mathrm{~s}^{-1}$. In such a close orbit, the matter captured by the NS as it moves along its elliptical orbit is able to produce a persistent X-ray emission exhibiting flux variability. This variability corresponds to the orbit eccentricity and to a periodical increase of the $N_{\mathrm{H}}$ column density when the NS is located close to the line connecting the observer and the supergiant. With this simple model, short flares cannot be explained; instead, abrupt transitions in the accretion rate should correspond to the wind clumping. Once the 


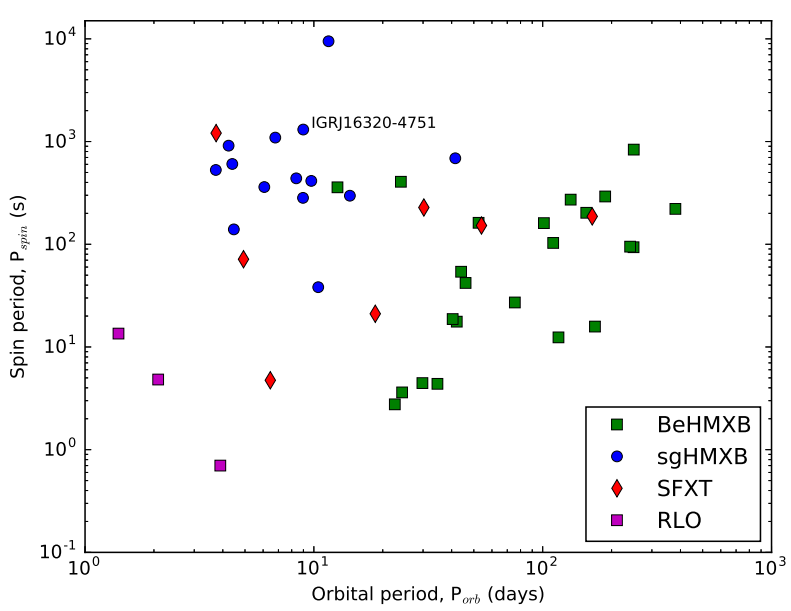

Fig. 7. Updated Corbet diagram (1986) showing the different populations of HMXBs (X-ray pulsars) with measured values of both $P_{\text {orb }}$ and $P_{\text {spin }}$. BeHMXB show a correlation between $P_{\text {orb }}$ and $P_{\text {spin }}$ due to net transfer of angular momentum between the decretion disk of the Be companion star and the neutron star. Beginning atmospheric Rochelobe overflow (RLO) systems have shorter (likely circularized) orbital periods. Supergiant fast X-ray transients (SFXT) are accreting pulsars exhibiting short and intense flares, spanning nearly the whole range of parameters of this diagram. Finally, supergiant HMXBs are accreting pulsars with quite short orbital periods (most being circularized) and slowly rotating neutron stars. IGR J16320-4751, labeled in this diagram, is one of these persistently accreting systems.

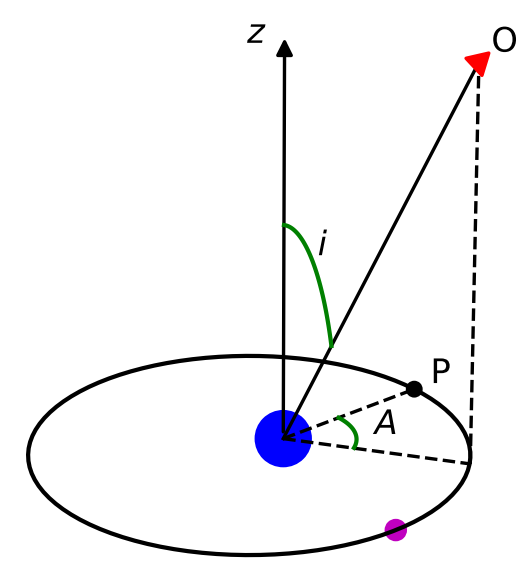

Fig. 8. Schematic view of our model. The neutron star (purple circle) describes an elliptical orbit in the $X Y$-plane around the supergiant star (blue circle). A distant observer $O$ (red triangle) is located in the direction defined by its angular coordinates $i$ (inclination) and $A$ (azimuth) with respect to the orbital plane and periastron position $P$.

stationary wind profile is defined, we still have three free parameters: the orbital eccentricity $e$, and the angular coordinates $A$ (azimuth) and $i$ (inclination) of a distant observer. These angles are measured with respect to the fixed orbital plane and periastron position $P$. A schematic view of our model can be seen in Fig. 8.

In order to compute the $N_{\mathrm{H}}$ column density, for each orbital phase (or time), we integrated the wind density profile along the line connecting the observer $O$ with the NS position. Moreover, we assumed that the Swift/BAT count rate is proportional to the local wind density at the position of the accreting NS. Under all the above mentioned assumptions, we simultaneously fitted the Swift/BAT folded light curve and the $N_{\mathrm{H}}$ phase evolu-
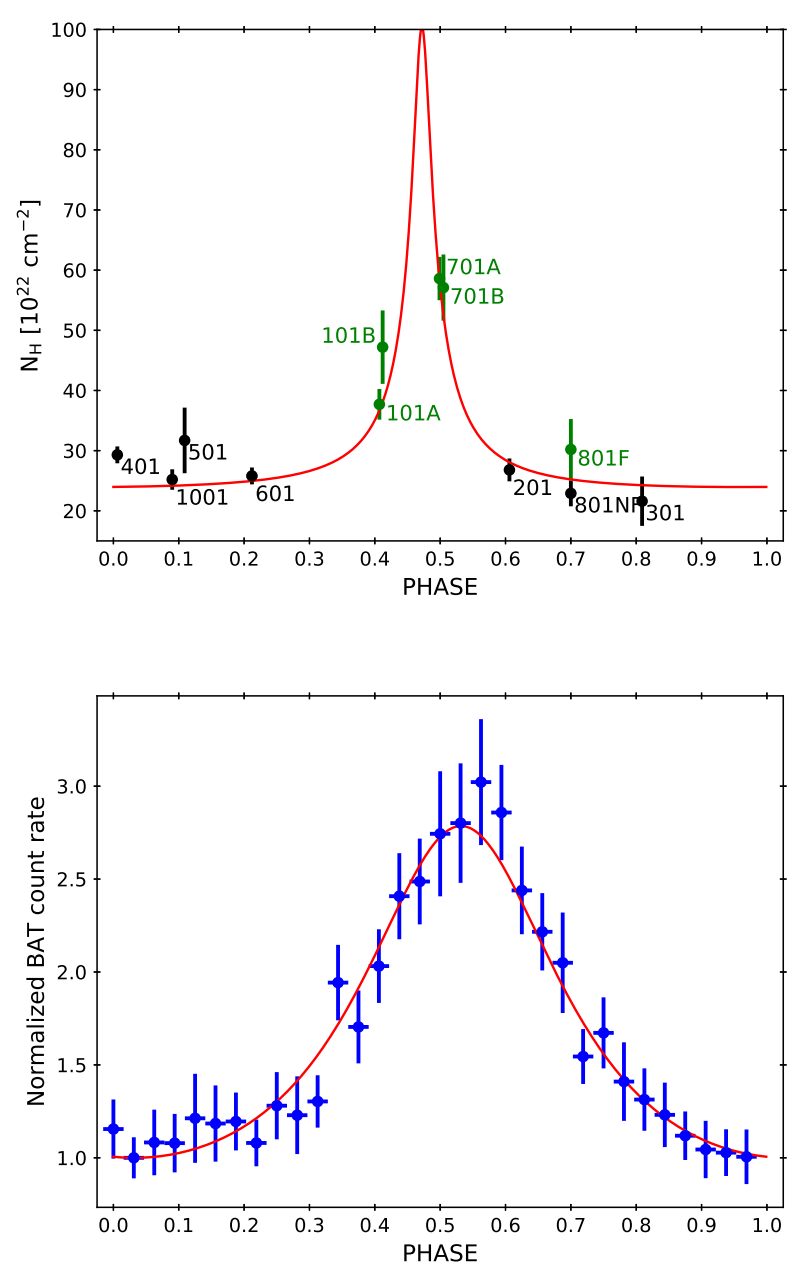

Fig. 9. Orbital evolution of IGR J16320-4751. Upper panel: intrinsic absorption column density, $N_{\mathrm{H}}$, obtained from XMM-Newton spectral fitting. Error bars correspond to the $90 \%$ confidence level. Lower panel: folded Swift/BAT light curve normalized by its minimum value. Error bars correspond to the 1- $\sigma$ confidence level. Red lines are the absorption column density and normalized local density of our simple wind model scenario obtained for the parameters indicated in the text.

tion obtaining an optimal solution given by $e=0.20 \pm 0.01$, $A=-146.3^{\circ}{ }_{-2.9}^{+3.7}$, and $i=62.1^{\circ}{ }_{-1.5}^{+0.3}$ (90\% confidence intervals) that we present in Fig. 9. The eccentricity is mainly constrained by the BAT light curve amplitude while the inclination depends strongly on the $N_{\mathrm{H}}$ increase profile. Our simple model is able to simultaneously fit both data sets, explaining the sudden change in the $N_{\mathrm{H}}$ column density, which peaks at a phase $\sim 0.47$, and the BAT smooth modulation, which has its maximum at a phase $\sim 0.53$. The phase difference between both maxima arises as a consequence of the observer azimuth angle.

The period uncertainty prevents us from accurately determining the phase of earlier XMM-Newton ObsIDs 0128531101 and 0201700301 , and thus we decided to avoid including them in the analysis of the orbital evolution. However, for the sake of the correlation of different spectral parameters independent of their orbital phase, these observations were fully incorporated in our analysis.

Regarding the $\mathrm{Fe} \mathrm{K} \alpha$ line, we were also able to recover a clear curve of growth as was previously found for other SGXBs sources, where the flux of the Gaussian normalized by the continuum flux is correlated with the $N_{\mathrm{H}}$ column (see right panel of Fig. 6). This suggests the existence of a strong link 
between the absorbing and the fluorescent matter that, together with the orbital modulation, points to the stellar wind as the main contributor to both continuum absorption and $\mathrm{Fe} \mathrm{K} \alpha$ emission in this source. Moreover, the narrowness of the Fe lines in all X-ray spectra allows us to confirm that IGR J16320-4751 belongs to the HMXB narrow-line group (Giménez-García et al. 2015).

\section{Conclusions}

In this paper we reported a comprehensive work on IGR J163204751, an archetype of the new class of highly absorbed HMXBs hosting supergiant stars, as shown by its localization in the Corbet diagram (Fig. 7). After having refined the orbital period, we investigated the geometrical and physical properties of both components of the binary system along their full orbit by analyzing phase-resolved X-ray spectra obtained with XMM-Newton and the hard X-ray light curve from Swift/BAT. First, we found a clear modulation of both the intrinsic hydrogen column density and the hard X-ray light curve with the orbital phase (Fig. 9). Second, we recovered two additional correlations: one connects the Fe $\mathrm{K} \alpha$ line flux to the column density, suggesting that fluorescent matter is related to absorbing matter, and the other relates the $\mathrm{Fe} \mathrm{K} \alpha$ line flux to the total continuum flux, suggesting that fluorescence emission emanates from a small region close to the accreting pulsar. Based on these two correlations we suggest that the absorbing matter is located within a small dense region surrounding the NS.

We then built a simple geometrical model of a NS orbiting a supergiant companion and accreting from its intense stellar wind. This simple model is able to reproduce the orbital modulation of both the sudden change in absorption column density and the smooth evolution of hard X-ray Swift/BAT folded light curve, as well as the phase shifts of their maxima. By putting together both correlations described above - the $\mathrm{Fe} \mathrm{K} \alpha$ line with column density and total continuum flux - and these two successful fits - the $N_{\mathrm{H}}$ and hard X-ray light curves - we unambiguously show that the orbital modulation of the three observed parameters - column density, hard X-ray flux, and the $\mathrm{Fe} \mathrm{K} \alpha$ line- is caused by intrinsic absorption of matter surrounding the NS, modulated by the stellar wind density profile as viewed by the observer along the line of sight.

To conclude, this work provides strong support to our current understanding of intrinsically absorbed sgHMXBs. Additional studies applied to the spectral evolution analysis, and in particular time-resolved and polarimetric X-ray observations sampled along their full orbit, would be of high value to better constrain the overall physical and geometrical properties of sgHMXBs.
Acknowledgements. We made use of the IGR source webpage maintained by J. Rodriguez (http://irfu.cea.fr/Sap/IGR-Sources). We are grateful to Francis Fortin for insightful discussions. FG and SC were partly supported by the LabEx UnivEarthS, Interface project I10, "From evolution of binaries to merging of compact objects." This work was partly supported by the Centre National d'Etudes Spatiales (CNES), and based on observations obtained with MINE: the Multi-wavelength INTEGRAL NEtwork. FG and JAC acknowledge support from PIP 0102 (CONICET). FAF is a fellow of CONICET. JAC is a CONICET researcher. This work received financial support from PICT-2017-2865 (ANPCyT). JAC was also supported on different aspects of this work by Consejería de Economía, Innovación, Ciencia y Empleo of Junta de Andalucía under excellence grant FQM-1343 and research group FQM-322, as well as FEDER funds.

\section{References}

Arnaud, K. A. 1996, in Astronomical Data Analysis Softwareand Systems V, ASP Conf. Ser., 101, 17

Castor, J. I., Abbott, D. C., \& Klein, R. I. 1975, ApJ, 195, 157

Chaty, S., \& Rahoui, F. 2012, ApJ, 751, 150

Chaty, S., Rahoui, F., Foellmi, C., et al. 2008, A\&A, 484, 783

Coleiro, A., Chaty, S., Zurita Heras, J. A., Rahoui, F., \& Tomsick, J. A. 2013, A\&A, 560, A108

Corbet, R. H. D. 1986, MNRAS, 220, 1047

Corbet, R., Barbier, L., Barthelmy, S. et al. 2005, ATel, 649

Foschini, L., Tomsick, J. A., Rodriguez, J., et al. 2004, 5th INTEGRAL Workshop on the INTEGRAL Universe, 552, 247

Giménez-García, A., Torrejón, J. M., Eikmann, W., et al. 2015, Highlights Spanish Astrophys. VIII, 482

Güver, T., \& Ozel, F. 2009, MNRAS, 400, 2050

in 't Zand, J. J. M., Ubertini, P., Capitanio, F., \& Del Santo, M. 2003, IAU Circ., 8077,2

Kallman, T. R., Palmeri, P., Bautista, M. A., Mendoza, C., \& Krolik, J. H. 2004 ApJS, 155, 675

Krimm, H. A., Holland, S. T., Corbet, R. H. D., et al. 2013, ApJS, 209, 14

Lebrun, F., Leray, J. P., Lavocat, P., et al. 2003, A\&A, 411, L141

Lutovinov, A., Rodriguez, J., Revnivtsev, M., \& Shtykovskiy, P. 2005, A\&A, 433, L41

Negueruela, I., \& Schurch, M. P. E. 2007, A\&A, 461, 631

Negueruela, I., Smith, D. M., Reig, P., Chaty, S., \& Torrejón, J. M. 2006, X-ray Univ. 2005, 604, 165

Oskinova, L. M., Feldmeier, A., \& Kretschmar, P. 2012, MNRAS, 421, 2820

Rahoui, F., Chaty, S., Lagage, P.-O., \& Pantin, E. 2008, A\&A, 484, 801

Rodriguez, J., Tomsick, J. A., Foschini, L., et al. 2003, A\&A, 407, L41

Rodriguez, J., Bodaghee, A., Kaaret, P., et al. 2006, MNRAS, 366, 274

Sguera, V., Bazzano, A., Bird, A. J., et al. 2006, ApJ, 646, 452

Strüder, L., Briel, U., Dennerl, K., et al. 2001, A\&A, 365, L18

Sugizaki, M., Mitsuda, K., Kaneda, H., et al. 2001, ApJS, 134, 77

Titarchuk, L. 1994, ApJ, 434, 570

Tomsick, J. A., Lingenfelter, R., Walter, R., et al. 2003, IAU Circ., 8076, 1

Turner, M. J. L., et al. 2001, A\&A, 365, L27

Ubertini, P., Lebrun, F., Di Cocco, G., et al. 2003, A\&A, 411, L131

Walter, R., Zurita Heras, J., Bassani, L., et al. 2006, A\&A, 453, 133

Wilms, J., Allen, A., \& McCray, R. 2000, ApJ, 542, 914

Winkler, C., Gehrels, N., Schönfelder, V., et al. 2003, A\&A, 411, L349

Zurita Heras, J. A., De Cesare, G., Walter, R., et al. 2006, A\&A, 448, 261

Zurita Heras, J. A., Chaty, S., Prat, L., \& Rodriguez, J. 2009, AIP Conf. Ser., 1126,313 